\title{
Stereoscopic determination of all-sky altitude map of aurora using two ground-based Nikon DSLR cameras
}

\author{
R. Kataoka ${ }^{1,2}$, Y. Miyoshi ${ }^{3}$, K. Shigematsu ${ }^{3}$, D. Hampton ${ }^{4}$, Y. Mori ${ }^{5}$, T. Kubo ${ }^{6}$, A. Yamashita $^{6}$, M. Tanaka ${ }^{7}$, \\ T. Takahei ${ }^{8}$, T. Nakai ${ }^{9}$, H. Miyahara ${ }^{10}$, and K. Shiokawa ${ }^{3}$ \\ ${ }^{1}$ National Institute of Polar Research, 10-3, Midoricho, Tachikawa, Tokyo 190-8518, Japan \\ ${ }^{2}$ Interactive Research Center of Science, Tokyo Institute of Technology, 2-12-1, Ookayama, Meguro-ku, Tokyo 152-8550, \\ Japan \\ ${ }^{3}$ Solar-Terrestrial Environment Laboratory, Nagoya University, Furo-cho, Chikusa-ku, Nagoya, Aichi 464-8601, Japan \\ ${ }^{4}$ Geophysical Institute, University of Alaska, Fairbanks, 903 Koyukuk Drive, Fairbanks, AK 99775-7320, USA \\ ${ }^{5}$ Department of Mechanical Engineering, Shizuoka University, 3-5-1 Johoku, Naka-ku, Hamamatsu, Shizuoka 432-8561, \\ Japan \\ ${ }^{6}$ Department of Precision Engineering, Graduate School of Engineering, The University of Tokyo, 7-3-1 Hongo, Bunkyo-ku, \\ Tokyo 113-8656, Japan \\ ${ }^{7}$ Department of Mechanical and Control Engineering, Tokyo Institute of Technology, 2-12-1 Ookayama, Meguro-ku, Tokyo \\ 152-8550, Japan \\ ${ }^{8}$ Orihalcon Technologies, Inc., Japan \\ ${ }^{9}$ Hydrospheric Atmospheric Research Center, Nagoya University, Furo-cho, Chikusa-ku, Nagoya, Aichi 464-8601, Japan \\ ${ }^{10}$ Musashino Art University, 1-736 Ogawa-cho, Kodaira City, Tokyo 187-8505, Japan \\ Correspondence to: R. Kataoka (kataoka.ryuho@nipr.ac.jp)
}

Received: 8 July 2013 - Revised: 2 August 2013 - Accepted: 3 August 2013 - Published: 6 September 2013

\begin{abstract}
A new stereoscopic measurement technique is developed to obtain an all-sky altitude map of aurora using two ground-based digital single-lens reflex (DSLR) cameras. Two identical full-color all-sky cameras were set with an $8 \mathrm{~km}$ separation across the Chatanika area in Alaska (Poker Flat Research Range and Aurora Borealis Lodge) to find localized emission height with the maximum correlation of the apparent patterns in the localized pixels applying a method of the geographical coordinate transform. It is found that a typical ray structure of discrete aurora shows the broad altitude distribution above $100 \mathrm{~km}$, while a typical patchy structure of pulsating aurora shows the narrow altitude distribution of less than $100 \mathrm{~km}$. Because of its portability and low cost of the DSLR camera systems, the new technique may open a unique opportunity not only for scientists but also for nightsky photographers to complementarily attend the aurora science to potentially form a dense observation network.
\end{abstract}

Keywords. Atmospheric composition and structure (airglow and aurora) - Ionosphere (auroral ionosphere) - Magnetospheric physics (energetic particles, precipitating)

\section{Introduction}

A number of beautiful pictures have been obtained using full-color digital single-lens reflex (DSLR) cameras around the world during this solar maximum. Recently, even astronomers onboard the international space station have taken tons of side-view images of aurora and airglow with Nikon DSLR cameras, which would be useful for the 3-D tomography of the upper atmosphere in the near future. It is also feasible that the emission altitude of detailed auroral structures can be estimated using the stereoscopic photographs taken by multiple ground-based cameras, because the DSLR cameras are now sensitive enough for aurora observations (ISO > 12 800), and the spatial resolution of more than several thousand pixels by several thousand pixels is several 
times higher than standard scientific-grade CCD cameras. These are the first scientific motivations of the Aurora 3D project (http://aurora3d.jp) which was originally a more outreach-oriented project to challenge 3-D dome projection by a minimum set of stereoscopic all-sky DSLR cameras. We conducted the Aurora 3-D experiment at Poker Flat Research Range, Alaska for three winter seasons in 2010-2011, 20112012, and 2012-2013.

Historically, about 100 years ago, Störmer (1913) first made the stereoscopic measurement of aurora to understand the basic form and height of aurora in detail. More recent trials of stereoscopic reconstruction of aurora were originated from the reconstruction of a luminous distribution by a scanning photometer chain in the magnetic meridian plane (Romick and Belon, 1967; Vallance Jones et al., 1991), and also from the altitude determination by triangulation using TV cameras (Stenbaek-Nielsen and Hallinan, 1979). Thereafter, Aurora Computed Tomography (ACT) was successful in reconstructing the 3-D distribution of auroral luminosity from monochromatic images obtained at multi-point stations at the same time (Aso et al., 1990, 1998; Frey et al., 1996; Nygrén et al., 1996). The inversion technique has been developed with the construction of ground-based camera networks such as the Aurora Large Imaging System (ALIS) (Steen and Brändström, 1993; Gustavsson, 1998). Tanaka et al. (2011) recently developed a new method of generalized ACT (GACT) that reconstructs the energy and spatial distributions of precipitating electrons from multi-instrument data. Suitably separated ground-based imaging systems, with a separation distance of several tens of $\mathrm{km}$, can now therefore be thought of as a single "meta-instrument" accessing a dimension which cannot be resolved through a single-site measurement.

On the other hand, in many former advanced studies (e.g., Kataoka et al., 2001; Ebihara et al., 2010), the emission altitude of aurora was usually assumed at a typical height of emission layer to investigate the meso-scale or global horizontal distribution to map an all-sky image on the geographic coordinates. Such a method of geographic transform has actually been effective, as validated by the partially overlapped patterns of observation data obtained from a number of allsky imagers installed at AGOs (automatic geophysical observatories) in Antarctica (Rosenberg and Doolittle, 1994) and GBOs (ground-based observatories) in Northern Hemisphere (Mende et al., 2008). However, an essential problem always remains that the emissions from a single ground-based allsky camera originated from many different altitudes at the same time, as can especially be seen in the ray structure. In other words, such an essential problem itself has important information of altitude profiles of small-scale aurora. Kubota et al. (1999) was the first who applied such a localized stereoscopic analysis on nightglow emissions. Ejiri et al. (2002) used the method of Kubota et al. (1999) to estimate the smallscale wave structures of nightglow emissions to investigate the generation mechanisms.
Our stereoscopic experiment focuses on the smallest scale structures of aurora, using a minimum set of instruments. We use vertically directed all-sky DSLR cameras with a separation distance of $8 \mathrm{~km}$ to achieve the fine spatial resolution of $1^{\circ}$, applying a straightforward method to estimate the emission altitude by correlating the apparent patterns of aurora within the corresponding localized pixels. The purpose of this paper is to report the first results of the "altitude map" as obtained from the all-sky stereoscopic analysis.

\section{Observations}

We installed two identical sets of a Nikon camera system at Poker Flat Research Range $\left(65.12^{\circ} \mathrm{N}, 147.43^{\circ} \mathrm{W}, 0.51 \mathrm{~km}\right.$ height from sea level) and Aurora Borealis Lodge $\left(65.04^{\circ} \mathrm{N}\right.$, $147.45^{\circ} \mathrm{W}, 0.73 \mathrm{~km}$ height). We refer to these camera systems as PI1 and PI2, respectively. The separation distance between PI1 and PI 2 is $8 \mathrm{~km}$, and is aligned approximately north-south. The camera system consists of Nikon D4 camera body equipped with a NIKKOR $8 \mathrm{~mm}$ F2.8 circular fisheye lens, controlled with a Windows PC laptop, which is connected to the internet to be remotely controlled even from Japan using the Nikon software of Camera Control Pro 2. The geographic position and the Universal Time are obtained from the GPS unit of Nikon GP-1. The original image size is 4928 pixels by 3280 pixels, and the 180 degrees all-sky circle determines 3192 pixels by 3192 pixels at the image center as shown in Figs. 1 and 2.

\section{Method of analysis}

First of all, we aligned the focus directions of the cameras using dozens of bright stars in the original images at PI1 and PI2, applying the method of Mori et al. (2013). The resultant "dome master" format is illustrated in Fig. 3a. In the dome-master format, geographic north is to the top, west is to the right, the center of images is the zenith, and the edge of the all-sky circle is at 90 degrees from the zenith. The radial distance from the center is linearly proportional to the angle from the zenith. The examples of obtained all-sky images in dome-master format are shown in Figs. 1 and 2.

Secondly, we remove the bright stars from the domemaster images by using a median filter of 20 pixels by 20 pixels for each R, G, and B image separately. Then we choose a small base window of 50 pixels by 50 pixels in a domemaster image of PI1, and we search the corresponding center pixels of the search window of the same size in the domemaster image of PI2, changing the assumed emission altitude from 50 to $400 \mathrm{~km}$ by a $10 \mathrm{~km}$ resolution. Here we set the maximum altitude of $400 \mathrm{~km}$ because an altitude of more than $400 \mathrm{~km}$ is too high to be accurately resolved by the $8 \mathrm{~km}$ separation distance. The altitude of the base window is finally determined by the assumed altitude of the highest normalized cross-correlation coefficient of the windows of PI1 


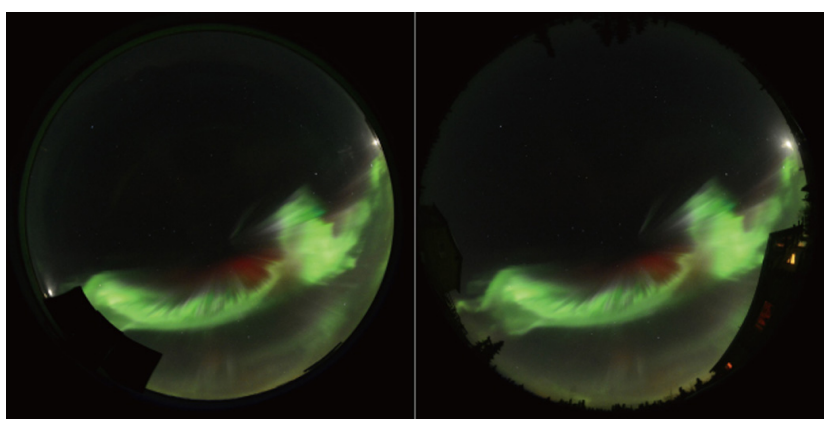

Fig. 1. All-sky images of ray structure of discrete aurora as obtained from (left) Poker Flat Research Range and from (right) Aurora Borealis Lodge at 09:17 UT on 17 March 2013. Camera settings are ISO6400 and $2 \mathrm{~s}$ exposure time. The center of images is the zenith, geographic north is to the top, and west is to the right.

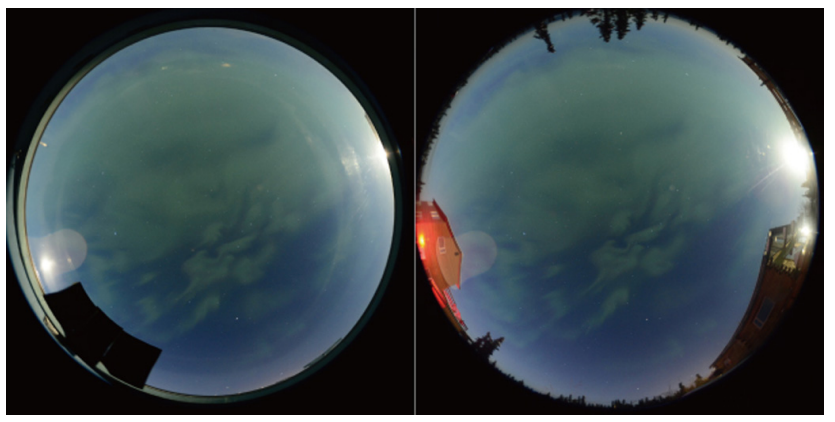

Fig. 2. All-sky images of patchy structure of pulsating aurora as obtained from (left) PFRR and from (right) ABL at 16:25 UT on 26 January 2013. Camera settings are ISO3200 and $5 \mathrm{~s}$ exposure time. The format is the same as Fig. 1.

and PI2 in R, G, or B images to make it robust for the selection of the color balance. The reason why we use the normalized cross-correlation of the "localized pattern" is also to make the method robust for using many different types of DSLR cameras of which the absolute luminosity or relative luminosity between the cameras cannot always be crosscalibrated. We only use the altitude where the maximum correlation coefficient was larger than 0.6. The base window is shifted half the size of the pixels of the base window (i.e., 25 pixels) to repeat the same sequence until filling the central 1500 by 1500 pixels to construct the altitude map.

A standard geographic coordinate transform is used to search for the corresponding pixels between the dome-master images of PI1 and PI2. The basic geographic coordinate system is illustrated in Fig. 3b. When we need to know the dome-master position of $\theta$ and $B$ corresponding to certain co-latitude $b$ and longitude $A$, we use the following set of (a) Dome Master Format

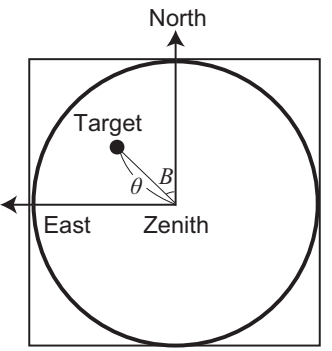

(b) Geographic Coordinate System

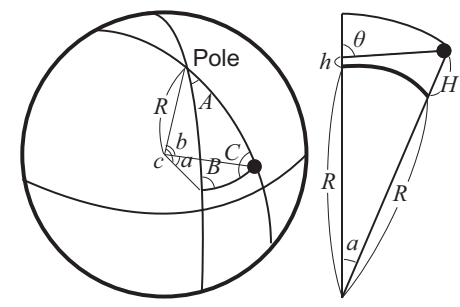

Fig. 3. Dome-master format (a) and corresponding geographic coordinate system (b), where $\theta$ is the zenith angle, $B$ is the azimuth angle from the north, and $A$ is the longitude between the target and observer. See the text for details.

equations:

$$
\begin{aligned}
\cos a & =\cos b \cos c+\sin b \sin c \cos A, \\
\cos B & =(\cos b \sin c-\sin b \cos c \cos A) / \sin a, \\
\tan \theta & =\frac{(R+H) \sin a}{(R+H) \cos a-(R+h)},
\end{aligned}
$$

where $c$ and $h$ are the co-latitude and altitude of the observation point, respectively, $H$ is the assumed emission altitude, and $R$ is the Earth's radius. On the other hand, when we need to know the co-latitude $b$ and longitude $A$ of a certain domemaster position of $\theta$ and $B$, we use the following set of equations:

$$
\begin{aligned}
\cos b & =\cos c \cos a+\sin c \sin a \cos B, \\
\sin A & =\sin B \sin a / \sin b, \\
\sin (\theta-a) & =\sin \theta(R+h) /(R+H) .
\end{aligned}
$$

The last step to complete the altitude map is to remove the potential noise values, assuming a continuity of the estimated altitudes at neighboring base windows. In this study, we remove the estimated altitude of a base window when any difference values from the neighboring windows are larger than $50 \%$ of the estimated altitude of the base window.

\section{Results}

The typical example of a ray structure of a discrete aurora is shown in Fig. 1, and the obtained altitude map with $1^{\circ}$ resolution (25 pixels) is shown in Fig. 4. It is generally known that red aurora is higher than green aurora, and the obtained altitude map clearly supports this tendency as shown in Fig. 4. Magnetic local midnight is about 11:00 UT, and this event occurred in the pre-midnight sector. This is a typical ray structure that appeared during a substorm during the main phase of a magnetic storm. The minimum Dst index of this magnetic storm was $-132 \mathrm{nT}$, which was one of the largest storms during the solar cycle 24 , and the amplitude of the associated substorm was also large, as indicated by the $\mathrm{AE}$ 

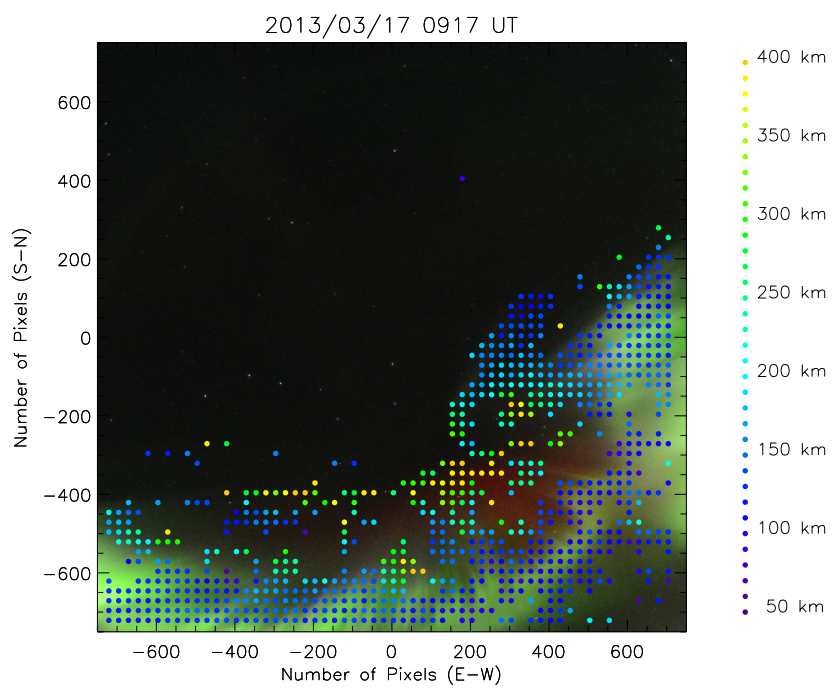

Fig. 4. Altitude map of a ray structure of a discrete aurora as obtained from all-sky images in Fig. 1. The superposed dots show the estimated emission altitudes, and the blue to yellow colors indicate low $(50 \mathrm{~km})$ to high $(>400 \mathrm{~km})$ altitudes. The full-color original image is also shown in the background.

index of around $1000 \mathrm{nT}$. This event occurred about $3 \mathrm{~h}$ after the arrival of interplanetary shock driven by a coronal mass ejection.

A typical example of a patchy structure of a pulsating aurora is shown in Fig. 2, and the obtained altitude map is shown in Fig. 5. It is generally known that pulsating auroras continue for several hours after a substorm expansion, especially post-midnight, associated with the precipitation of energetic electrons with higher characteristic energies than those of other types of aurora (Brown et al., 1976). The relatively low altitudes as shown in Fig. 5 are consistent with the expected higher energies of precipitating electrons. This event belongs to a weak magnetic storm with a minimum Dst of $-51 \mathrm{nT}$, which was driven by a high-speed solar wind flowing from a coronal hole (e.g., Kataoka and Miyoshi, 2006).

Figure 6 shows the occurrence distributions of altitudes for both ray structure and patchy structure as shown in Figs. 4 and 5 , respectively. It is clearly seen that the typical altitude of ray structure is about $100-130 \mathrm{~km}$, with broad distributions of higher altitude up to $250 \mathrm{~km}$. On the other hand, it is also clearly seen that the typical altitude of the patchy aurora is $80-90 \mathrm{~km}$, with relatively narrow distributions against the height. These results are consistent with previous reports on the altitude distributions that depend on types of aurora (Brown et al., 1976). It is noted that the stopping height of $4 \mathrm{keV}$ electrons is $\sim 110 \mathrm{~km}$, while that for $30 \mathrm{keV}$ electrons is $\sim 90 \mathrm{~km}$, and therefore different altitudinal profiles are results of different energy spectra of precipitating electrons.

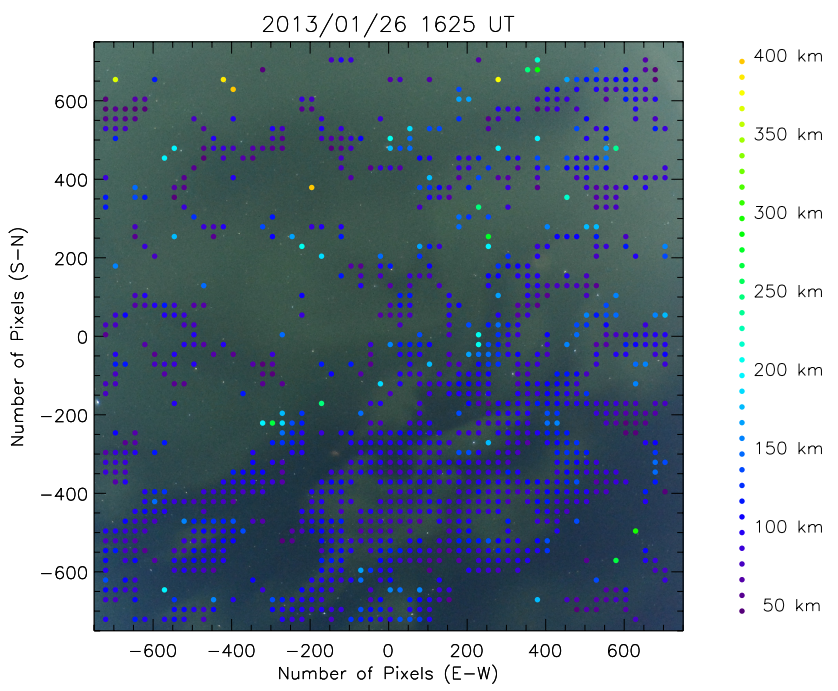

Fig. 5. Altitude map of a patchy structure of a pulsating aurora as obtained from all-sky images in Fig. 2. The format is the same as Fig. 4.
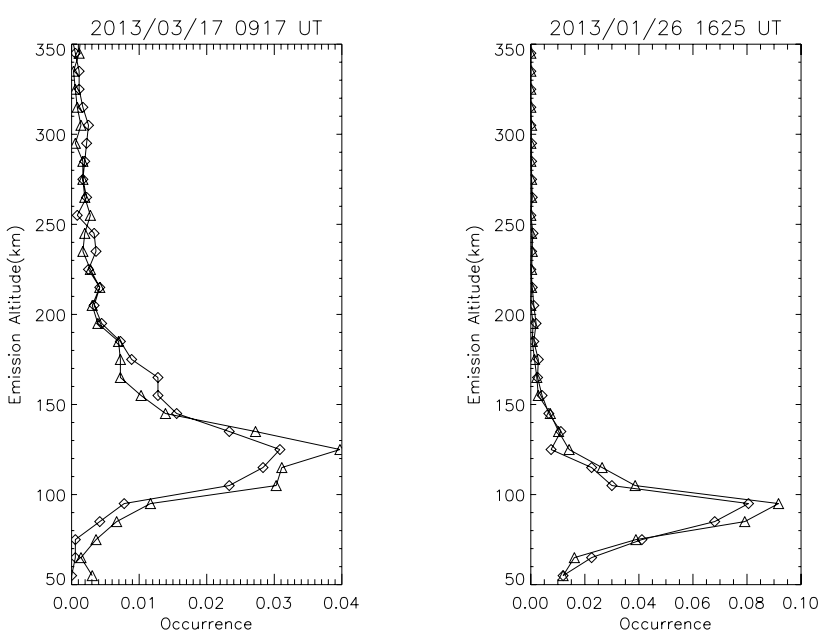

Fig. 6. The occurrence distributions of the emission altitudes for (left) the ray structure, and for (right) the patchy structure, as also shown by the dots in Figs. 4 and 5, respectively. Diamonds are from the altitude map as calculated starting from the base window in PI1, and triangles are from the altitude map as calculated starting from the base window in PI2.

\section{Summary and discussions}

In summary, we developed a new measurement technique to obtain an all-sky altitude map of auroras with a minimum set of instruments of two all-sky DSLR cameras. The estimated altitude maps of the ray and patchy structure are reasonable as shown in Figs. 4-6, and are consistent with former observations and theories.

The largest error to estimate the altitude is originated from the deformation of the corresponding windows, which 
depends on the location of the corresponding center pixels of the search window at dome-master images. The window size should therefore not be too large to make a significant deformation error. However, for example, in the top half of Fig. 5, it became difficult to match the windows against the diffuse aurora over a very broad area, which is significantly larger than the window size. Another intrinsic error originated from the assumption of only one value of emission altitude within the base window. To avoid the error, we should set the window size as small as possible, but the window size should not be too small to identify the corresponding patterns. Such a trade-off tuning for selecting the best size of the windows will improve the accuracy. Considering these potential errors, we checked the errors by repeating the same analysis in both directions, i.e., setting the base window in PI1 or in PI2, and obtained essentially the same results as shown in Fig. 6 by diamonds and triangles.

The PI1 was put in a room under a large acrylic dome of diameter of $1.2 \mathrm{~m}$, and the PI 2 was put inside a small enclosure of 30-foot acrylic dome, and both cameras were not put at the exact center of the acrylic dome. The "dome deformation" effect should be calibrated first by checkerboard patterns etc. This calibration procedure was omitted in this paper and causes potential errors, especially closer to the edge. This is one reason we do not use the data closer to the edge, and we focus only on the central part. Such a basic calibration would enlarge the spatial range of the altitude map, and also improve the accuracy of altitude estimation.

This is a first step to prove the feasibility of the method of analysis of all-sky altitude map, and to demonstrate the potential of low-cost DSLR cameras for stereoscopic observations. Considering the portability and the low cost, dozens of multi-point observations may have a great potential as a new meta-instrument. Combination with other measurements would of course be a powerful tool. The obtained results are encouraging, not only for scientists but also for nightsky photographers to contribute to the advancement of solarterrestrial physics. This study was conducted by using only commercial products. Although we used the best cameras and best lenses for this experiment, all of the equipment can be replaced by other affordable cameras and lenses to fit their own purpose without significantly reducing the performance. For example, the whole system approximately costs several thousand dollars today.

Further, constructing side-by-side dome-master images for a standard 3-D projection, we can observe the 3-D aurora movies by our eyes, with the help of 3-D projectors and computer software of Amateras Media Player (http://orihalcon. jp/amaterasplayer/). Such an attempt may lead to significant new findings, especially by auroral researchers, and it is also useful for outreach purpose for scientists. Further, it is important for making a great opportunity of using circular fish-eye lenses for observations of many purposes. Such an attempt will be reported at some point in the future.
Acknowledgements. R. Kataoka thanks Toshikazu Ebisuzaki for giving the idea to initiate the Aurora 3-D project, and we thank Ayumi Yaegashi, Takanori Nishiyama, Takeshi Sakanoi, Kazushi Asamura, and Yoko Fukuda for their help maintaining the project. We thank Mok Kumagai for his kind help installing the latest camera system at Aurora Borealis Lodge. R. Kataoka thanks Hirobumi Suzuki, Mio Nishiyama, Hidehiko Tanaka, and Tomonao Harada for providing Nikon cameras and NIKKOR lenses, and for their helpful technical support. R. Kataoka thanks Yoshimasa Tanaka, Mitsumu Ejiri, and Yasunobu Ogawa of National Institute of Polar Research for their careful reading of this paper to improve the discussions. This work was carried out by the joint research program of the Solar-Terrestrial Environment Laboratory. The Aurora 3-D project had been supported by Housou Bunka Foundation 2011-2012. This work was in part supported by KAKENHI, Grant-in-Aid for Challenging Exploratory Research (25540114).

Topical Editor K. Hosokawa thanks one anonymous referee for her/his help in evaluating this paper.

\section{References}

Aso, T., Hashimoto, T., Abe, M., Ono, T., and Ejiri, M.: On the analysis of aurora stereo observations, J. Geomag. Geoelectr., 42, 579-595, 1990.

Aso, T., Ejiri, M., Urashima, A., Miyaoka, H., Steen, A., Brändström, U., and Gustavsson, B.: First results of auroral tomography from ALIS-Japan multi-station observations in March, 1995, Earth Planets Space, 50, 81-86, 1998.

Brown, N. B., Davis, T. N., Hallinan, T. J., Stenbaek-Nielsen, H. C.: Altitude of pulsating aurora determined by a new instrumental technique, Geophys. Res. Lett., 3, 403-404, 1976.

Ebihara, Y., Kataoka, R., Weatherwax, A. T., and Yamauchi, M.: Dayside proton aurora associated with magnetic impulse events: South Pole observations, J. Geophys. Res., 115, A04301, doi:10.1029/2009JA014760, 2010.

Ejiri, M. K., Shiokawa, K., Ogawa, T., Kubota, M., Nakamura, T., and Tsuda, T.: Dual-site imaging observations of small-scale wave structures through $\mathrm{OH}$ and OI nightglow emissions, Geophys. Res. Lett., 29, 10, doi:10.1029/2001GL014257, 2002.

Frey, S., Frey, H. U., Carr, D. J., Bauer, O. H., and Haerendel, G.: Auroral emission profiles extracted from three-dimensionally reconstructed arcs, J. Geophys. Res., 101, 21731-21741, 1996.

Gustavsson, B.: Tomographic inversion for ALIS noise and resolution, J. Geophys. Res., 103, 26621-26632, 1998.

Kataoka, R. and Miyoshi, Y.: Flux enhancement of radiation belt electrons during geomagnetic storms driven by coronal mass ejections and corotating interaction regions, Space Weather, 4, S09004, doi:10.1029/2005SW000211, 2006.

Kataoka, R., Fukunishi, H., Lanzerotti, L. J., Maclennan, C. G., Frey, H. U., Mende, S. B., Doolittle, J. H., Rosenberg, T. J., and Weatherwax, A. T.: Magnetic Impulse Event: A detailed case study of extended ground and space observations, J. Geophys. Res., 106, 25873-25889, 2001.

Kubota, M., Ishii, M., Shiokawa, K., Ejiri, M. K., and Ogawa, T.: Height measurements of nightglow structures observed by allsky imagers, Adv. Space. Res., 24, 593-596, 1999.

Mende, S. B., Harris, S. E., Frey, H. U., Angelopoulos, V., Russell, C. T., Donovan, E., Jackel, B., Greffen, M., and Peticolas, 
L. M.: The THEMIS Array of Ground-based Observatories for the Study of Auroral Substorms, Space Sci. Rev., 141, 357-387, doi:10.1007/s11214-008-9380-x, 2008.

Mori, Y., Yamashita, A., Tanaka, M., Kataoka, R., Miyoshi, Y., Kaneko, T., Okutomi, M., and Asama, H.: Calibration of FishEye Stereo Camera for Aurora Observation, International Workshop on Advanced Image Technology (IWAIT2013), Proceedings of the International Workshop on Advanced Image Technology (IWAIT2013), 729-734, 2013.

Nygrén, T., Markkanen, M., Lehtinen, M., and Kaila, K.: Application of stochastic inversion in auroral tomography, Ann. Geophys., 14, 1124-1133, doi:10.1007/s00585-996-1124-1, 1996.

Romick, G. J. and Belon, A. E.: The spatial variation of auroral luminocity-II, Planet. Space Sci., 15, 1695-1716, 1967.

Rosenberg, T. J. and Doolittle, J. A.: Studying the polar ionosphere and the magnetopause with automatic geophysical observatories: The U. S. program in Antarctica, Antarct. J. U. S., 29, 347-349, 1994.

Steen, $\AA$. and Brändström, U.: ALIS-A multi-station ground-based imaging system at high latitudes, STEP International Newsletter, 3, 11-14, 1993.
Stenbaek-Nielsen, H. C. and Hallinan, T. J.: Pulsating auroras: Evidence for non-collisional thermalization of precipitating electrongs, J. Geophys. Res., 84, 3257-3271, 1979.

Störmer, C.: ON an Auroral Expedition to Bossekop in the Spring of 1913, Astrophys. J., 38, 311-314, 1913.

Tanaka, Y.-M., Aso, T., Gustavsson, B., Tanabe, K., Ogawa, Y., Kadokura, A., Miyaoka, H., Sergienko, T., Brändström, U., and Sandahl, I.: Feasibility study on Generalized-Aurora Computed Tomography, Ann. Geophys., 29, 551-562, doi:10.5194/angeo29-551-2011, 2011.

Vallance Jones, A., Gattinger, R. L., Creutzberg, F., Harris, F. R., McNamara, A. G., Yau, A. W., Llewellyn, E. J., Lummerzheim, D., Rees, M. H., McDade, I. C., and Margot, J.: The ARIES auroral modelling campaign: Characgerization and modelling of an evening auroral arc observed from a rocket and a ground-based line of meridian scanners, Planet. Space Sci., 39, 1677-1705, 1991. 